\title{
Pacing in a Woman with Full Term Pregnancy: First Case Report in Bangladesh
}

\author{
AW Chowdhury, ${ }^{1}$ MA Muttalib, ${ }^{1}$ AEM Mazharul Islam, ${ }^{2}$ AK Choudhury, ${ }^{1}$ M Faruk, ${ }^{2}$ K Begum, ${ }^{3}$ AB \\ Siddiq, ${ }^{1}$ S Dutta, ${ }^{1}$ RS Giasuddin, ${ }^{1}$ DK Mondol, ${ }^{1}$ AHM Kamal, ${ }^{1}$ M Shakawatullah, ${ }^{1}$ \\ ${ }^{1}$ Department of Cardiology, National Institute of Cardiovascular Diseases, Dhaka. ${ }^{2}$ Department of \\ Cardiology, Dhaka Medical College, Dhaka. ${ }^{3}$ Department of Obstetrics and Gynecology, Dhaka Medical \\ College, Dhaka.
}

\begin{abstract}
Key Words: Congenital complete heart block, Pregnancy, Pacemaker.

Abstract:

A 19-year-old primigravida was diagnosed to have complete heart block (CHB) during her antenatal checkup at 36 weeks. The patient had history of syncope a few years back. She was referred to the National Institute of Cardiovascular Diseases (NICVD). A temporary pacemaker was implanted at NICVD. She was then referred to Dhaka Medical College Hospital (DMCH), where a baby girl was delivered by cesarean section. A permanent pacemaker was implanted later at NICVD. To our knowledge this is the first case report of pace maker implantation in a pregnant woman from Bangladesh.
\end{abstract}

(Cardiovasc. j. 2009; 1(2) : 237-239)

\section{Introduction:}

Congenital complete atrioventricular block (CCAVB) is a relatively rare and potentially serious problem in pregnancy. ${ }^{1}$ There have been relatively few reports of complete heart block in pregnancy. Obstetric experience in women who had pacemakers implanted before conception has been reported, ${ }^{2}$ but there is a paucity of information on the management of complete heart block detected for the first time during pregnancy or labour. The patients of CCAVB with pregnancy create a special category because of the inherent risk associated with stress of normal vaginal delivery or cesarean section. Labour in these patients may be complicated by syncope and convulsions caused by slowing of the heart rate during the Valsalva maneuver exercised at the time of forceful contractions of the second stage of labour. ${ }^{3}$ Moreover a pregnant woman cannot readily undergo permanent pacemaker implantation because of the radiation hazard involved with fluoroscopic procedure. We are reporting a case of CCAVB with full term pregnancy that has been managed by the coordinated action of cardiologist and obstetrician of two separate hospitals.

\section{Case Report:}

A 19-year-old lady (Fig.1), hailing from Madaripur, presented with complaints of occasional palpitation and full term pregnancy. She had a history of sudden fall on the ground 4 years back at school. She also experienced a syncopal attack 3 years ago while taking bath in a pond. Her antenatal period was uneventful. An ECG was done as part of her pre-operative assessment (for cesarean section) and it showed complete heart block with ventricular rate 43 beats/ min. (Fig.2). The patient did not have history of taking any beta-blocker, calcium-channel blocker or digitalis. She was referred to National Institute of Cardiovascular Diseases (NICVD). On examination her pulse rate was 38 beats/ minute, regular and blood pressure was 110/60mm Hg. Mild pallor was present. Otherwise her general, physical and cardiovascular examinations were unremarkable. On abdominal examination, there was a single foetus in cephalic presentation and uterine height corresponded to 36 weeks of gestation. There were mild uterine contractions

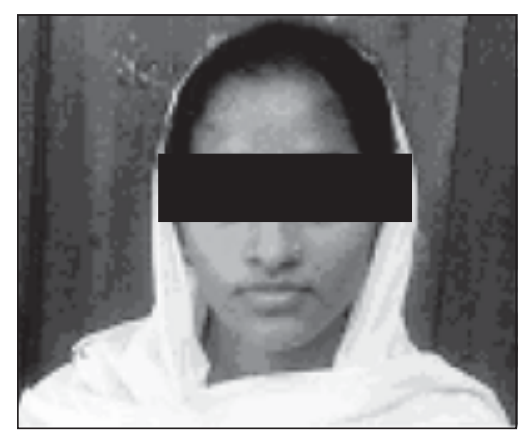

Fig.-1. The patient Mrs. Pervin

Address of Correspondence- Dr Abdul Wadud Chowdhury, Associate Professor of Cardiology, National Institute of Cardiovascular Diseases, Dhaka, Bangladesh 


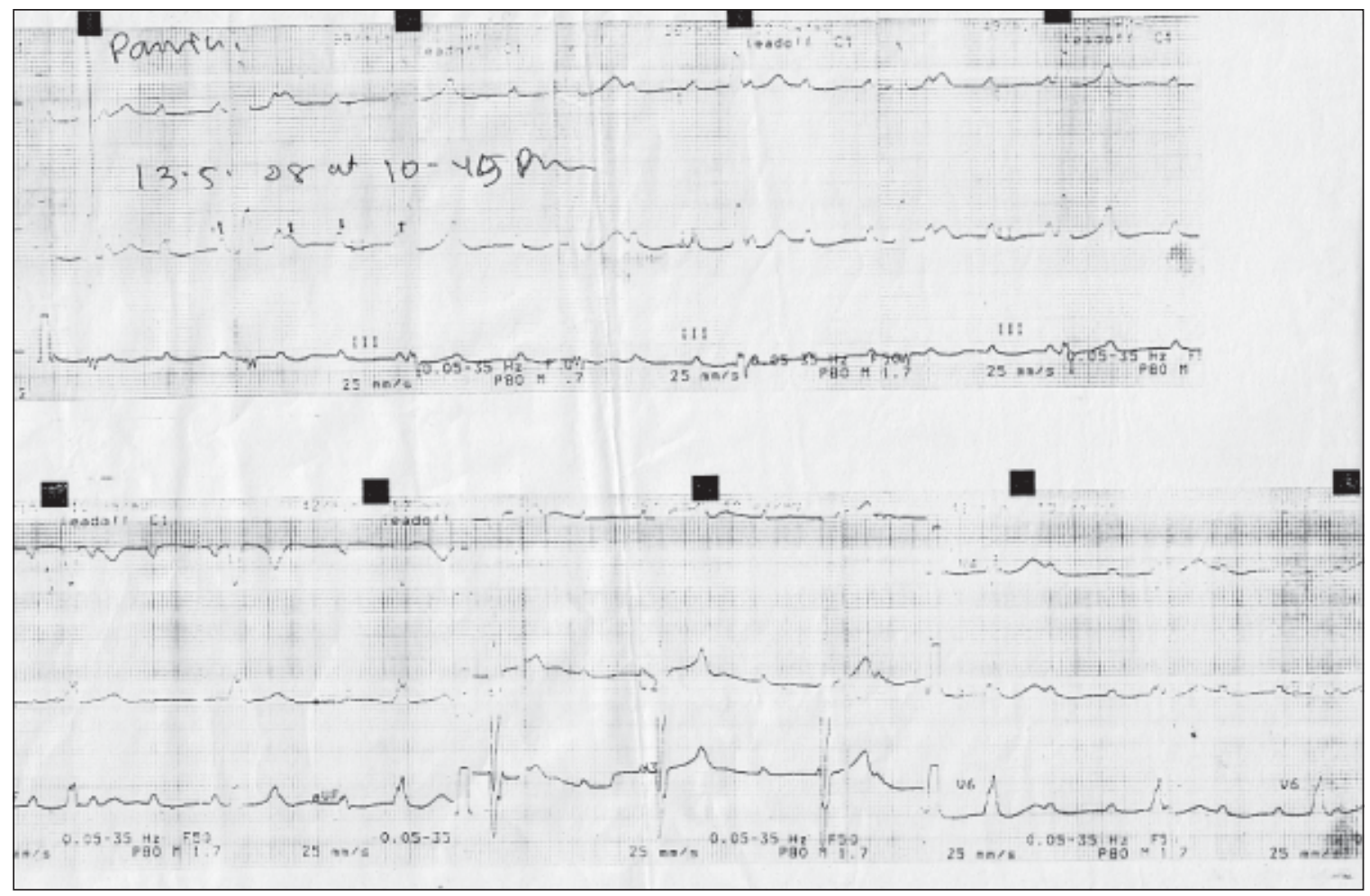

Fig.-2: ECG showing complete heart block with a ventricular rate of about $43 / \mathrm{min}$.

$\&$ foetal heart rate was 140 beats/minute. Echocardiography was normal. The decision to perform a cesarean section was taken along with arrangements for a temporary pacemaker. A temporary pacemaker was inserted through the right femoral vein with pacing set at 60 beats/ minute. She was then transferred to Dhaka Medical College Hospital (DMCH) and a cesarean section was done on the same day under spinal anesthesia. She gave birth to a healthy female child. Her postpartum period was uneventful. One week later she was transferred back to NICVD and a single chamber permanent pacemaker (VVI) was implanted. Her postoperative period was uneventful and she was discharged from the hospital in a stable and satisfactory condition after 10 days. At follow-up after six weeks she was found quite all right and symptom free.

\section{Discussion:}

Complete heart block described during pregnancy is mostly of congenital origin. ${ }^{4}$ Congenital complete heart block (CCAVB) is a rare disease with an incidence of 1 in 20,000 live births. ${ }^{5}$ The aetiology is not completely understood yet. Most often patients with congenital complete heart block are asymptomatic. However the onset of symptoms in patients with CCAVB can also occur later in life. This is due to a variable degree of heart block and heart rate. Most of the symptoms are related to the slow heart rate resulting in hydrops foetalis, heart failure of the neonate or exercise intolerance of the child. Longer pauses may cause presyncope, syncope in the form of classical Stoke-Adams attack, or even sudden cardiac death. These symptoms are present in patients with resting heart rate of 50 beats/min or less. Prolonged recovery times of escape foci following rapid pacing, slow heart rates on 24-hour ECG recordings and occurrence of paroxysmal tachycardia may be the predisposing factors to the development of symptoms. ${ }^{6}$

Mortality from congenital complete heart block is highest in the neonatal period, is much lower in childhood and adolescence and increases slowly later in life. However, it is difficult to predict prognosis in an individual patient. ${ }^{6}$ Rarely, CCAVB resolves spontaneously. In most patients, the degree of conduction abnormality will either persist or worsen over time. ${ }^{7,8}$ The young asymptomatic patients with CCAVB who have a mean daytime heart rate of more than 50 beats/ minute do not require treatment. ${ }^{6}$ However 
patients with symptomatic bradyarrythmia should receive treatment. ${ }^{6}$

Sustained bradycardia may be encountered during pregnancy for a number of reasons. Electrolyte imbalance such as hyperkalemia, metabolic derangements such as hypothyroidism, or medications such as beta-blockers, calcium-channel blockers, or digitalis are the easily remediable causes of bradycardia during pregnancy. ${ }^{9}$ Congenital complete heart block presenting for the first time in pregnancy is a therapeutic challenge. Management involves use of cardiac pacemaker in symptomatic patient, which should be implanted whenever heart block is diagnosed in pregnancy to maintain cardiac function. However, prophylactic placement of a pacemaker is not indicated in asymptomatic patients. ${ }^{9,} 10$ For symptomatic patients in the first and second trimesters, permanent pacemaker implantation is the therapy of choice; it can be performed under echocardiographic control. ${ }^{4,}{ }^{11}$ In symptomatic patients, who present at or near term, temporary pacing followed by the induction of labour at the earliest possible time is suggested, to prevent complications of prolonged temporary pacing. ${ }^{9}$ Overall maternal and neonatal outcome is unaffected in such cases.

In our case the malady is identified at full term pregnancy with quite a low heart rate (38 beats/ $\mathrm{min}$ ) and with previous history of syncope. Planned caesarean section was carried out under temporary pacing coverage and later a permanent pacemaker was implanted resulting in excellent symptom free status. To our knowledge this is the first such case report from Bangladesh where close cooperation between cardiologist and obstetrician of two hospitals has resulted in a happy ending by successful implantation of pacemaker in a pregnant woman.

\section{References}

1. Sullivan JM, Ramanathan KB. Management of medical problems in pregnancy- severe cardiac disease. $N$ Engl J Med 1985; 313: 304-309

2. Jaffe R, Gruber A, Fejgin M, Altaras M, Ben-Aderet N. Pregnancy with an artificial pacemaker. Obstet Gynecol Surv 1987; 42: 137-139

3. Schonbrun M, Rowland W, Quiroz AC. Complete heart block in pregnancy. Successful use of an intravenous pacemaker in 2 patients during labor. Obstet Gynecol 1966; 27: 243-246

4. Lau CP, Lee CP, Wong CK, Cheng CH, Leung WH. Rate responsive pacing with a minute ventilation sensing pacemaker during pregnancy and delivery. Pacing Clin Electrophysiol 1990; 13: 158-163

5. Michaelsson M, Allen Engle M, Congenital complete heart block: an international study of the natural history. Cardiovasc Clinics 1972;4:85-101.

6. Douglas P. Zipes. Specific Arrhythmias: Diagnosis and Treatment.in: Braunwald E, editor. Heart disease. $5^{\text {th }}$. ed. New york; W B Saunders Company; 1997.

7. Esscher EB. Congenital complete heart block in adolescence and adult life. A follow up study. Eur Heart J 1981;2:281-288.

8. Michaelsson M, Jonzon A, Riesenfeld T. Isolated complete atrioventricular block in adult life, a prospective study. Circulation 1995;92:442-449

9. Kenmure ACF, Cameron AJV. Congenital complete heart block in pregnancy. Br Heart $J$ 1976; 29: 911-913

10. Dalvi BV, Chaudhuri A, Kulkarni HL, Kale PA. Therapeutic guidelines for congenital complete heart block presenting in pregnancy. Obstet Gynecol 1992; 79: 802-804

11. Gudal M, Kervancioglu C, Oral D, Gurel T, Erol C, Sonel A. Permanent pacemaker implantation in a pregnant woman with the guidance of ECG and twodimensional echocardiography. Pacing Clin Electrophysiol 1987; 10: 543-545 